\title{
High gradient quadrupoles for low emittance storage rings
}

\author{
G. Le Bec, J. Chavanne, C. Benabderrahmane, L. Farvacque, L. Goirand, \\ S. Liuzzo, P. Raimondi, and F. Villar \\ ESRF-The European Synchrotron, 38000 Grenoble, France \\ (Received 29 February 2016; published 9 May 2016)
}

\begin{abstract}
High gradient quadrupoles are key components for the coming generation of storage ring based light sources. The typical specifications of these magnets are: almost $100 \mathrm{~T} / \mathrm{m}$ gradient, half a meter long, and a vertical aperture for the extraction of the x-ray beam. This paper presents the preparation work done at the European Synchrotron Radiation Facility, from the design to the manufacture and measurements of a prototype. It demonstrates the feasibility of such magnets. Different aspects of magnet engineering are discussed, including the study of the main scale factors and the preliminary design, the pole shaping, the impact of mechanical errors, and the magnetic measurements of a prototype with a stretched-wire system.
\end{abstract}

DOI: 10.1103/PhysRevAccelBeams.19.052401

\section{INTRODUCTION}

Projects of very high brightness, storage ring-based synchrotron light sources have been launched around the world [1-3]. In all of these projects the beam emittance is decreased by using a large number of low field dipole magnets, typically seven bends in each arc instead of two or three in the storage rings of the previous generation. These new storage rings must fit existing complexes, including accelerator tunnel and beam lines. The gradient of the strongest quadrupoles of the European Synchrotron Radiation Facility-Extremely Brilliant Source (ESRFEBS) and of the Advanced Photon Source Upgrade will be close to $100 \mathrm{~T} / \mathrm{m}$.

The ESRF is a $6 \mathrm{GeV}$ light source located in Grenoble, France, built in the early 1990's. The brilliance of the ESRF $\mathrm{x}$-ray source is dominated by the horizontal emittance of the electron beam, which is determined by the lattice of the storage ring. The emittance of the present ESRF storage ring is $4 \mathrm{~nm}$ rad in the horizontal plane and $5 \mathrm{pm} \cdot \mathrm{rad}$ in the vertical plane; the ESRF-EBS project aims to reduce the horizontal emittance to $135 \mathrm{pm} \cdot \mathrm{rad}$. The new ESRF storage ring should be commissioned in 2020 .

A schematic view of one ESRF-EBS cell is given in Fig. 1. The storage ring is composed of 32 cells, i.e. almost 1100 magnets will be built and installed. The ESRF-EBS magnets, including "exotic" dipoles, are briefly described in [4]. The present paper is dedicated to the design of the QF6 and QF8 high gradient quadrupole magnets, with gradients of approximately $90 \mathrm{~T} / \mathrm{m}$.

\footnotetext{
*lebec@esrf.fr

Published by the American Physical Society under the terms of the Creative Commons Attribution 3.0 License. Further distribution of this work must maintain attribution to the author(s) and the published article's title, journal citation, and DOI.
}

The maximum values of the gradient have been chosen to be compatible with conventional, iron dominated, normal conducting magnet technology. The bore radius of the ESRF-EBS high gradient quadrupoles is $r_{0} \approx 12.5 \mathrm{~mm}$, which gives an equivalent pole tip field $B_{\text {eq }}=r_{0} G \approx 1.1 \mathrm{~T}$ with $G=90 \mathrm{~T} / \mathrm{m}$. For comparison, the highest equivalent pole tip field in the present ESRF ring is $22 \mathrm{~T} / \mathrm{m} \times 33 \mathrm{~mm} \approx 0.7 \mathrm{~T}$, and the pole tip field of the high gradient quadrupoles installed in the European XFEL is $100 \mathrm{~T} / \mathrm{m} \times 8 \mathrm{~mm} \approx 0.8 \mathrm{~T}$.

This "moderate" pole tip field does not mean the poles are not saturated. Indeed, they are saturated and their magnetic polarization is above $1.9 \mathrm{~T}$. One may question the use of normal conducting magnets, instead of superconductors or permanent magnets. Up to now, superconductor magnets are almost absent in light source storage rings (except for a few insertion devices), because of the high heat load of these machines due to synchrotron

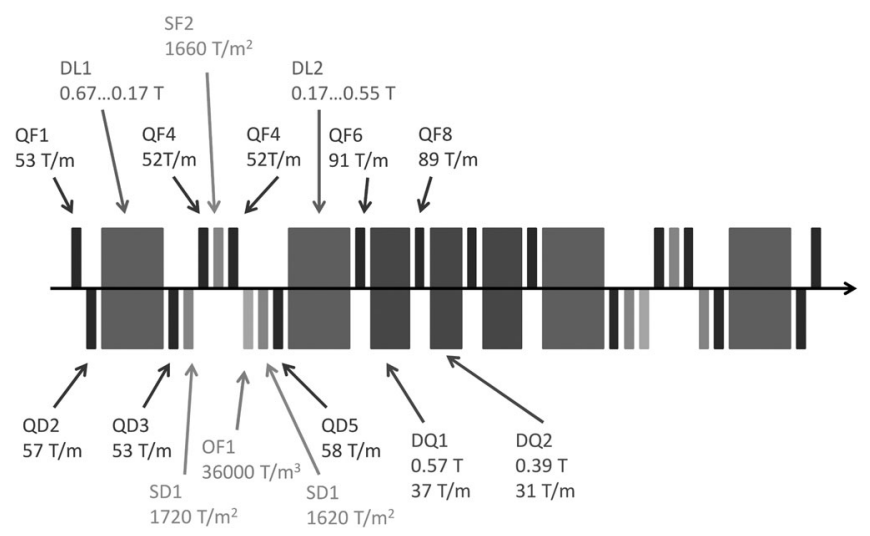

FIG. 1. Schematic view of one cell of the ESRF-EBS lattice. The storage ring will be made of 32 cells. QF, QD: quadrupoles; SF, SD: sextupole; OF: octupole magnets; DL: dipole with longitudinal gradient; DQ: dipole-quadrupoles. The fast correctors are not represented. 
TABLE I. Maximum systematic values of the field multipoles (defined in Appendix) expressed at $7 \mathrm{~mm}$.

\begin{tabular}{lc}
\hline \hline$n$ & $b_{n}$ \\
\hline 6 & $1 \times 10^{-4}$ \\
10 & $0.5 \times 10^{-4}$ \\
\hline \hline
\end{tabular}

radiation. Permanent magnets, on the other hand, are very suitable for compact, small aperture, high field designs, but suffer from a reduced tuning range and a decreased field quality in the case the magnets are not iron dominated [5]. Moreover, all of the magnets should be delivered in 2018: the time scale is short and not compatible with the development of permanent magnet solutions.

The field quality was specified on a $7 \mathrm{~mm}$ radius good field region (GFR). The maximum values of the field harmonics are given in Table I. These values are for the systematic multipoles coming from the magnet design.

All of the magnets in the ESRF-EBS storage ring shall be compact: the iron-free length of each arc is $5 \mathrm{~m}$ and there is only $15 \mathrm{~cm}$ free per magnet for fitting the coil ends, the beam position monitors, the bellows of the vacuum chambers, etc. The integration length of the magnets should be shortened as much as possible by a careful design of the coils.

The power consumption is another important design criteria for these magnets. The overall power consumption of the ESRF storage ring should be reduced, and the magnet system is a major contributor to the power losses. The reduction of the power consumption is achieved by designing the coils for low current densities, which has an impact on the compactness of the magnets.

The magnet design is detailed in Sec. II. The scale factors and a preliminary design are presented first (Sec. II B), then the pole shapes are optimized for improving the field quality and reducing the magnetic errors vs current (Secs. II C and II D). The impact of the mechanical errors on the random multipole errors and on the beam dynamics is discussed in Sec. II E. The influence of the fringe field terms on the electron beam is briefly looked at in Sec. II F, as a matter of curiosity. A high gradient quadrupole prototype has been built. The results of the prototype measurements are given in Sec. III.

\section{MAGNET DESIGN}

\section{A. Simulation tools}

The RADIA magnet simulation software is used for the field computation. The RADIA software was developed in the ESRF Insertion Device lab and has been largely used for undulator, wiggler and multipole magnet design [6-11]. This software does not rely on a finite element method as most of the field computation codes do, but on a boundary integral method. The magnets are described as a set of field sources, i.e. magnetization or currents. In the present version of RADIA, elementary magnetized objects have a uniform magnetization and are modeled as equivalent magnetic charges. The magnetic field and its integral along a straight line can be computed for each field source, at any point, using analytical formulas.

\section{B. Scale factors and preliminary design}

Water-cooled accelerator magnets are commonly operated at a moderate current density $J=5 \mathrm{~A} / \mathrm{mm}^{2}$; this value results from a compromise between power consumption and magnet compactness. The magnet bore radius is determined by the vacuum chamber and the physical aperture. Then, a rough approximation of the gradient is given by

$$
G \approx \frac{2 \mu_{0} N I}{r_{0}^{2}},
$$

$\mu_{0}$ being the magnetic permeability of the vacuum, $N$ the number of conductor turns, $I$ the current and $r_{0}$ the magnet bore radius; Eq. (1) is derived from the Ampère theorem, assuming an infinite permeability in the iron, and is no longer valid for the high gradient, nearly saturated quadrupole described in the next paragraphs. The gradient can also be obtained from the conservation of the magnetic flux in the pole (Fig. 2). Assuming the pole is saturated, one obtains

$$
\int_{0}^{X_{0}} G x d x \approx B_{\mathrm{Sat}} w
$$

where the notation $B_{\text {Sat }}=\mu_{0} M_{\text {Sat }}$ is for the saturated magnetic polarization. The so-called demagnetizing field, which opposes the magnetization, is neglected here. If the pole has a hyperbolic shape, the gradient is

$$
G \approx \frac{g}{\sqrt{2} r_{0}{ }^{4}}\left(2 r_{0}^{2}-g^{2}\right) B_{\text {Sat }} .
$$

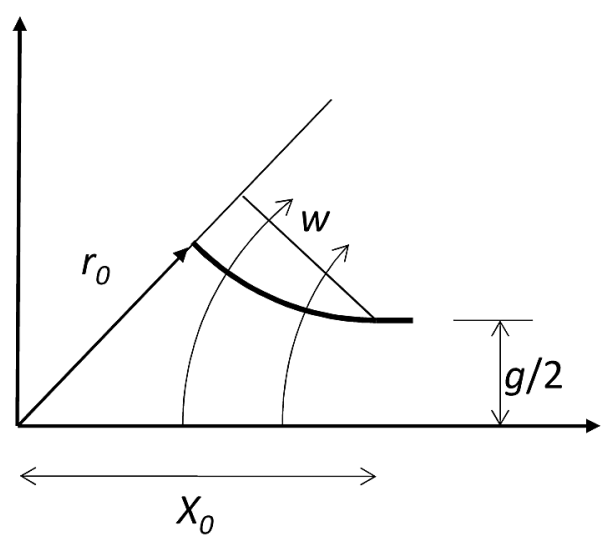

FIG. 2. Conservation of the magnetic flux across the pole. 
The gradient reaches its maximum value at $g \approx \sqrt{2 / 3} r_{0}$ :

$$
G_{\mathrm{Sat}} \approx \frac{4}{3 \sqrt{3}} \frac{B_{\text {Sat }}}{r_{0}}
$$

Equations (1) and (4) give

$$
\begin{aligned}
(N I)_{\mathrm{Sat}} & \approx \frac{2 r_{0}}{3 \sqrt{3}} M_{\mathrm{Sat}} \\
& \approx \frac{8}{27} \frac{\mu_{0} M_{\mathrm{Sat}}{ }^{2}}{G_{\mathrm{Sat}}} .
\end{aligned}
$$

It is well known that increasing the gradient implies reducing the magnet apertures. Equation (5) shows that high gradient magnets require less current than lower gradient, larger aperture magnets; the saturated field is simply reached at lower current for a smaller bore radius.

The power consumption of the four quadrupole coils of width $w$, height $h$, length $L$ and filling factor $\lambda$ is

$$
\begin{aligned}
P & \approx 8 \rho w h L J^{2} \lambda \\
& \approx \frac{16 r_{0} M_{\text {Sat }}}{3 \sqrt{3}} \rho L J \\
& \approx \frac{64}{27} \frac{\mu_{0} M_{\text {Sat }}^{2}}{G_{\text {Sat }}} \rho L J,
\end{aligned}
$$

where $\rho$ is the resistivity of the conductor. For a given iron length and total length of the magnet, the length of the coil heads, i.e. the coil width, is fixed: any extra turn would lead to longer coils. The outer radius of the magnet scales linearly with the height $h$ of the coils: $r_{\text {OUT }}=r+h$ where $r \approx r_{0}+r_{2} \cos \left(\pi / 4-\theta_{2}\right)+r_{5}$ represents the bore radius, the radial position of the coils and the thickness of the magnet yoke (see Fig. 3). At constant saturation field the outer radius writes

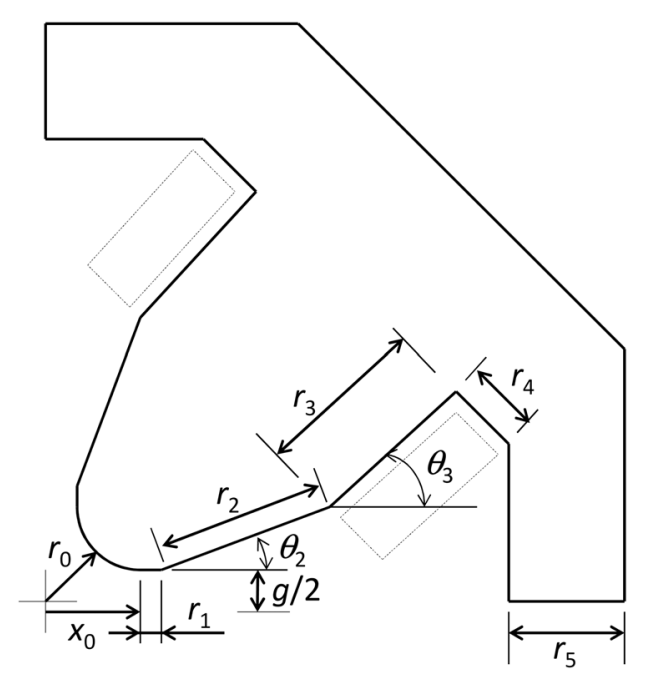

FIG. 3. Definition of the main geometrical magnet parameters.

$$
r_{\mathrm{OUT}} \approx r+\frac{8}{27} \frac{\mu_{0} M_{\mathrm{Sat}}^{2}}{G_{\mathrm{Sat}} w \lambda J} \approx r_{1}+\frac{512}{729} \frac{\mu_{0}^{2} M_{\mathrm{Sat}}^{4} \rho L}{G_{\mathrm{Sat}}{ }^{2} w \lambda P} .
$$

Equations (6) and (7) shows that: (i) the power consumption scales with the current density $J$, the radius and the saturation magnetization, (ii) the outer radius of the magnet scales with $1 / J$, (iii) the outer radius scales with $1 / P$. The current density results from a compromise between the power consumption and the transverse dimensions of the magnet, which has an impact on the mechanical integration, on the mass, on the mechanical resonant frequencies, etc.

The field quality suffers from the reduction of the magnet aperture, leading to higher multipole content for a given mechanical tolerance (the multipole content is defined in the Appendix). Assuming a small geometrical error $\varepsilon$, the $N$ th multipole field error $\Delta B_{N}$ (expressed at the bore radius) varies as

$$
\begin{aligned}
\Delta B_{N}\left(r_{0}\right) & \propto \varepsilon / r_{0} \\
& \propto \varepsilon G / B_{\text {Sat }} .
\end{aligned}
$$

At a given GFR radius, the $N$ th multipole error on the GFR boundary scales with

$$
\Delta B_{N}(r)=\left(\frac{r}{r_{0}}\right)^{N-1} \Delta B_{N}\left(r_{0}\right)
$$

The accuracy of the pole shape and the magnet assembly is technologically limited. Equations (8) and (9) show that for a given production technology, high gradient, low aperture quadrupoles are subject to higher multipole content than moderate gradient magnets.

Let us now study the impact of the main magnet geometrical parameters on the magnet performances. Until the end of this section, we will present the preliminary design of a $44 \mathrm{~T}$ integrated field gradient magnet, with a $12.5 \mathrm{~mm}$ bore radius, and a $11 \mathrm{~mm}$ gap between poles.

At constant integrated field gradient, a shorter magnet shall produce a higher gradient. If the magnet saturates, the excitation current increases much faster than the gradient and the power consumption may become extremely high. This effect is difficult to express with a simple analytical equation because of the nonlinear shape of the $M(H)$ curve of the material, but can be checked easily with any simulation software. This is shown in Fig. 4(a). The prototype is operated at a slightly saturated working point; it would be possible to design a shorter length, higher gradient quadrupole at the price of an increased power consumption. The mass of the magnet passes through a minimum [Fig. 4(b)]. For shorter length, the Ampère turns increase rapidly, leading to a large magnet cross section. 

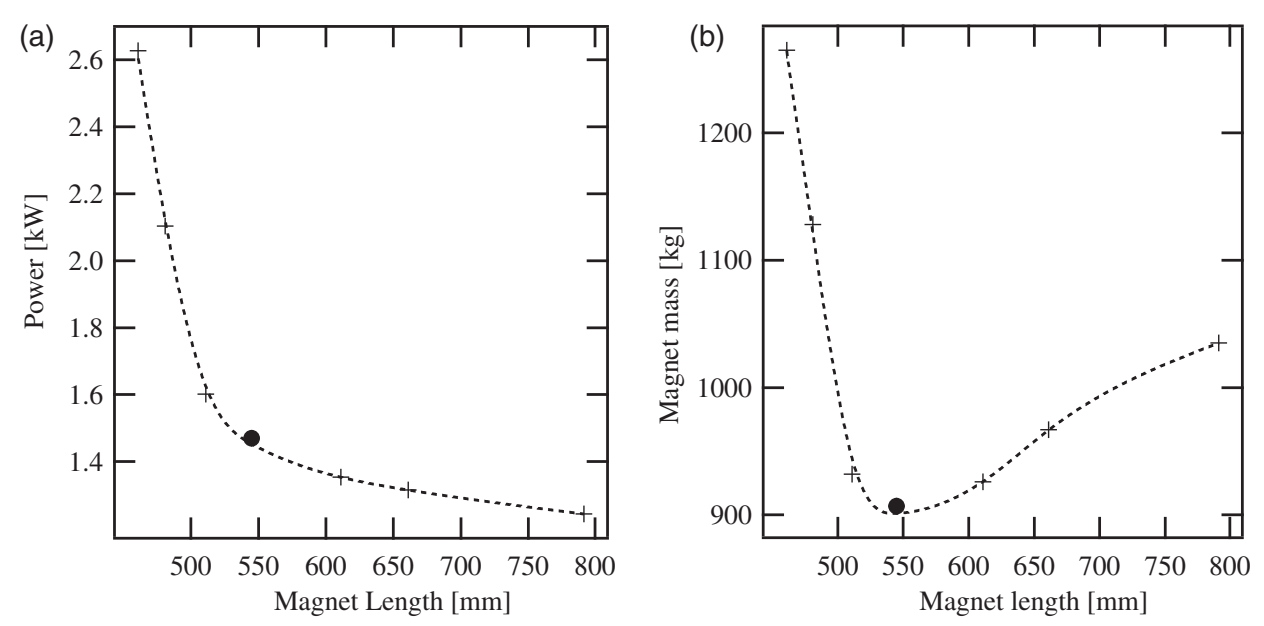

FIG. 4. Magnet power consumption (a) and mass (b) vs total length (including coil heads), at $44 \mathrm{~T}$ integrated field gradient and $12.5 \mathrm{~mm}$ bore radius. The crosses are 3D simulation results and the black disk indicates the parameters of the prototype magnet. The lines are spline interpolations between the simulated points.

It is useful to introduce the magnetic efficiency

$$
\eta=\frac{\int G d s}{P}
$$

where $s$ is the longitudinal coordinate. The pole taper, defined by the angle $\theta_{2}$ and the radius $r_{2}$, impacts on the magnetic efficiency. Let us start by varying the taper angle $\theta_{2}$, while keeping constant $r_{2}=80 \mathrm{~mm}$. Figure 5 shows the magnetic efficiency for different taper angles. At low taper angles, $\theta_{2}<10^{\circ}$, the magnetic efficiency is degraded by the strong flux leakage between the poles, which are close to each other all along the taper. At large taper angles, $\theta_{2}>30^{\circ}$, the saturation of the poles is stronger due to their reduced cross section, so the

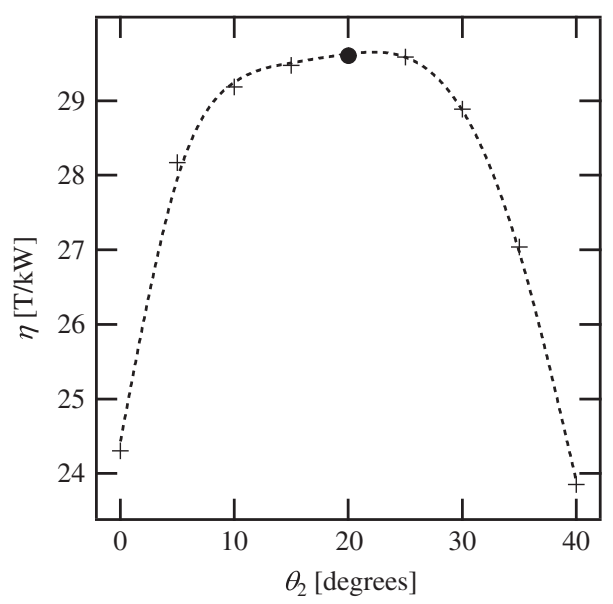

FIG. 5. Magnetic efficiency $\eta$ as defined in Eq. (10), vs taper angle $\theta_{2}$, at $r_{0}=12.5 \mathrm{~mm}, r_{2}=80 \mathrm{~mm}$ and for a $545 \mathrm{~mm}$ total length (including coil heads). The black dot indicates the design value and the lines are spline interpolations between the simulated points (markers). magnetic efficiency goes down. A moderate taper angle $\theta_{2}=20^{\circ}$ has been chosen, leading to an almost optimized efficiency. If the angle is set to this optimum value, the taper radius $r_{2}$ has a small impact on the efficiency.

Figure 6 shows the magnetic efficiency vs the coil taper angle, for different values of the pole taper radius $r_{2}$. The magnetic efficiency passes through a maximum value which depends on the pole taper radius. For large pole taper radius, the optimum value is reached at $\theta_{3}=45^{\circ}$, i.e. for a straight coil. The efficiency is reduced by only 2 percent when the pole taper radius is increased from 40 to $80 \mathrm{~mm}$. The value of $r_{2}$ has been set to $80 \mathrm{~mm}$ to facilitate the insertion of the electron beam vacuum chamber.

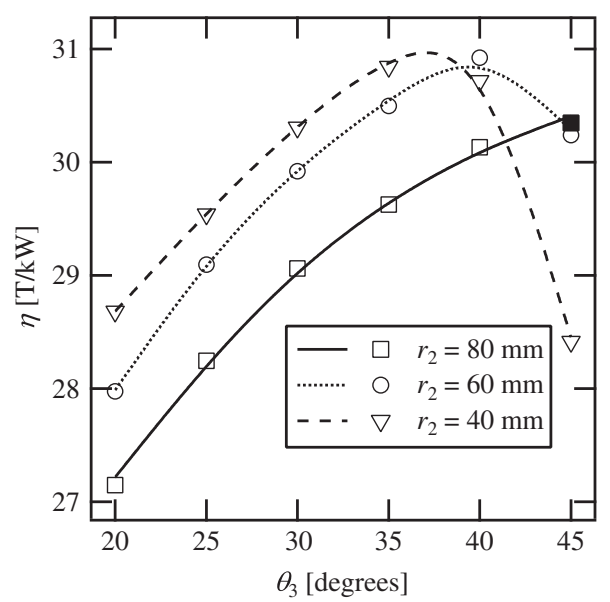

FIG. 6. Magnetic efficiency $\eta$ vs coil taper angle $\theta_{3}$, for different values of the pole taper length $r_{2}$, at $\theta_{2}=20^{\circ}$. The black square indicates the design value and the lines are spline interpolations between the simulated points (markers). 


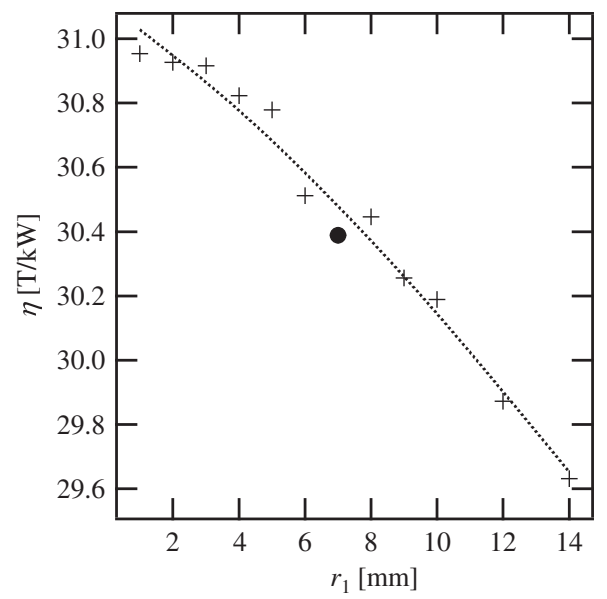

FIG. 7. Magnetic efficiency $\eta$ vs $r_{1}$ pole radius. The dot indicates the design value and the line is a spline interpolation between the simulated points (markers).

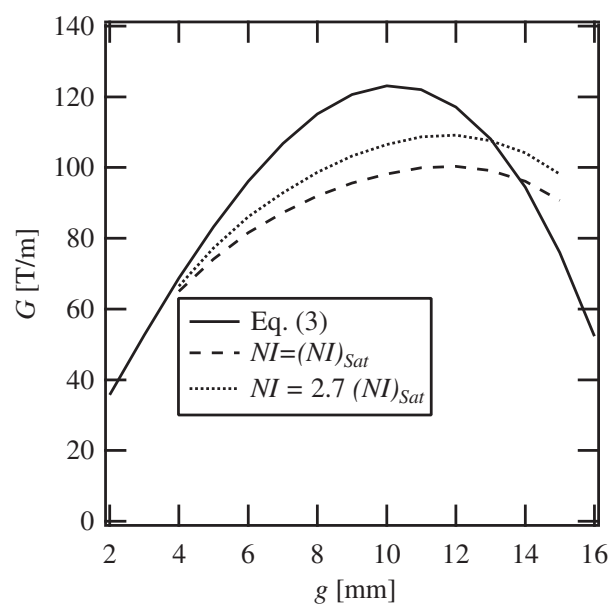

FIG. 8. Gradient vs gap between poles obtained from Eq. (3) and with a $3 \mathrm{D}$ model with Table III parameters $\left(r_{1}=1 \mathrm{~mm}\right.$ for the simulations shown in this plot). $(N I)_{\text {Sat }}=7668 \mathrm{~A}$ turns. The current density is $3.7 \mathrm{~A} / \mathrm{mm}^{2}$ at $(N I)_{\text {Sat }}$ and $10 \mathrm{~A} / \mathrm{mm}^{2}$ at $2.7(N I)_{\text {Sat }}$. The bore radius is $12.5 \mathrm{~mm}$.

The radius $r_{1}$ has a small impact on the magnetic efficiency (Fig. 7). Increasing slightly the $r_{1}$ radius facilitates the quality control of the magnet assembly.

The main design parameters of the high gradient quadrupoles are given in Table II. The coil of the prototype magnet is tapered with a $20^{\circ}$ angle. The larger $r_{1}$ radius of the serial magnets allows the design of a straight coil. The parameters $r_{3}$ and $r_{4}$ are not critical for the optimization of the magnetic efficiency. They are a function of the number of coil turns and coil layers. The number of coil layers is limited by the required longitudinal compactness of the magnet: any additional layer increases the insertion length. The number of coil layers has been set to four, leading to a $484 \mathrm{~mm}$ iron length, $545 \mathrm{~mm}$ long magnet. The coil design relies on basic electrical engineering and is not presented in detail in this paper. The main coil parameters are shown in
TABLE II. Design parameters of the high gradient quadrupoles. The prototype magnet has a tapered coil, while the series magnets will have a straight coil.

\begin{tabular}{lcc}
\hline \hline Type & Proto & Series \\
\hline$r_{0}$ & $12.5 \mathrm{~mm}$ & $12.5 \mathrm{~mm}$ \\
$g$ & $11 \mathrm{~mm}$ & $11 \mathrm{~mm}$ \\
$x_{0}$ & $14 \mathrm{~mm}$ & $14 \mathrm{~mm}$ \\
$r_{1}$ & $7 \mathrm{~mm}$ \\
$r_{2}$ & $3 \mathrm{~mm}$ & $80 \mathrm{~mm}$ \\
$\theta_{2}$ & $80 \mathrm{~mm}$ & 20 degrees \\
$r_{3}$ & 20 degrees & $147 \mathrm{~mm}$ \\
$\theta_{3}$ & $132 \mathrm{~mm}$ & 45 degrees \\
$r_{4}$ & 35 degrees & $41 \mathrm{~mm}$ \\
$r_{5}$ & $41 \mathrm{~mm}$ & $75 \mathrm{~mm}$ \\
Iron length & $75 \mathrm{~mm}$ & $484 \mathrm{~mm}$ \\
Magnetic length & $484 \mathrm{~mm}$ & $493 \mathrm{~mm}$ \\
Total length & $493 \mathrm{~mm}$ & $545 \mathrm{~mm}$ \\
Material & $545 \mathrm{~mm}$ & AISI 1006 \\
\hline \hline
\end{tabular}

Table III. The low power of the magnet is driven by the reduced current density. According to Eq. (6), the same magnet would dissipate $2.9 \mathrm{~kW}$ at $5 \mathrm{~A} / \mathrm{mm}^{2}$ current density and $5.8 \mathrm{~kW}$ at $10 \mathrm{~A} / \mathrm{mm}^{2}$.

Let us compare the parameters in Table III and the results obtained from Eqs. (1) to (7). The analytical model leads a maximum gradient of $122 \mathrm{~T} / \mathrm{m}$ and $1.9 \mathrm{~kW}$ at $N I=(N I)_{\text {Sat }}=7668 \mathrm{~A} \cdot$ turns, while the 3D model gives a $99 \mathrm{~T} / \mathrm{m}$ gradient and a power of $2.5 \mathrm{~kW}$ at the same excitation. (The parameters in Table III are given at the nominal excitation $N I=7007$ A.turns. The conversion to $N I=(N I)_{\text {Sat }}$ is straightforward.)

Figure 8 shows the evolution of the gradient with the gap between poles, according to Eq. (3) and from a 3D model. As already indicated and as expected, the gradient obtained from the analytical expression is overestimated. The gradient passes through a maximum, as predicted by Eq. (3). The optimum gap is $10.2 \mathrm{~mm}$ for the analytical model and $12 \mathrm{~mm}$ for the 3D model. It should be mentioned here that the homogeneity of the field gradient is expected to be better at small gaps. The correction of the field errors induced by the severe truncation of the poles is discussed in the next section.

TABLE III. Coils and electrical parameters.

\begin{tabular}{lc}
\hline \hline Nominal current & $91 \mathrm{~A}$ \\
Number of turns & 77 \\
Conductor layers & 4 \\
Conductor sections & $6 \times 6 \mathrm{~mm}^{2}$ \\
Cooling pipe diameter & $3 \mathrm{~mm}$ \\
Current density & $3.25 \mathrm{~A} / \mathrm{mm}^{2}$ \\
Temperature rise & $16 \mathrm{~K}$ \\
Magnet resistance & $0.24 \Omega$ \\
Magnet inductance & $0.21 \mathrm{H}$ \\
Magnet power & $2 \mathrm{~kW}$ \\
\hline \hline
\end{tabular}


The magnet outer radius can be computed with Eq. (7) and the magnet parameters, leading to a $280 \mathrm{~mm}$ magnet radius at $3.25 \mathrm{~A} / \mathrm{mm}^{2}$, a $240 \mathrm{~mm}$ radius at $5 \mathrm{~A} / \mathrm{mm}^{2}$ and a $200 \mathrm{~mm}$ radius at $10 \mathrm{~A} / \mathrm{mm}^{2}$, assuming a $\lambda=0.66$ coil filling factor. The width of the prototype magnet is $606 \mathrm{~mm}$, which is close to the $2 \times 280 \mathrm{~mm}$ estimation given by Eq. (7).

\section{Pole shaping algorithm}

In the previous paragraphs, the magnet has been optimized disregarding the quality of the magnetic field, i.e. the homogeneity of the integrated gradient and the spurious field harmonics (field harmonics and associated notations are defined in Appendix). For practical reasons, a $11 \mathrm{~mm}$ vertical gap between poles is needed for the integration of the electron beam vacuum chamber. This vertical gap has a strong effect on the field quality. Considering a $7 \mathrm{~mm}$ radius good field region (GFR) and a $\rho_{0}=7 \mathrm{~mm}$ reference radius, the gradient inhomogeneity reaches $1.8 \%$ and the systematic 12-poles reaches $32 \times 10^{-4}$ of the quadrupole component without specific pole shaping. These values are 1 order of magnitude above the specifications.

Finding a pole shape that optimizes the homogeneity of the integrated field is a so-called inverse problem. Different pole shapes may lead to almost the same field quality. Even if it is possible to design simple shims by manually varying one or two parameters on a magnet model, it is much more efficient to use a pole shaping algorithm.

Magnet optimization algorithms have been developed for a few decades and have been applied to optimize the homogeneity of particle accelerator magnets, nuclear resonance and magnetic resonance imaging magnets [12-15]. At the ESRF, an important effort has been made to improve the time efficiency of magnetic field computations and shape optimization $[8,16]$.

The pole shaping algorithm is briefly described below (the interested reader may find more details in the conference paper [16]). The main parts of the algorithms are the cost function, which depends on the field quality and other constraints (gradient strength, vertical gap between the poles, etc.), the parameters of the magnet models, and the optimization routine, which is based on a regularized descent method. As the optimization procedure starts with a fairly satisfactory magnet, it is assumed that finding the local minimum of a cost function is sufficient. This assumption is of prior importance because it allows us to restrict our study to descent methods and to exclude time consuming metaheuristics like simulated annealing or genetic algorithms.

\section{Cost function}

The 2D multipoles introduced in Appendix are suitable for the expression of the field quality. One can define a target multipole vector $\mathbf{C}_{0}=\left(0, \rho_{0} \int G d s, 0, \ldots 0\right)$ where $\rho_{0}$ is the radius of the good field region. The multipole errors are given by $\boldsymbol{\varepsilon}=\mathbf{C}-\mathbf{C}_{0}=\mathbf{M}_{\mathbf{C}}{ }^{+} \mathbf{B}-\mathbf{C}_{0}$.

Without any constraint on the vertical gap between poles, the algorithm tends to use all the space available: this may lead to a closure of the pole gap. This issue can be corrected by implementing a barrier function with high values if the gap is below the specifications or low values in the other case. In practice, it was implemented by an exponential function and stacked to the multipole errors:

$$
\varepsilon=\left(\mathbf{M}_{\mathbf{C}}{ }^{+} \mathbf{B}-\mathbf{C}_{0}, \alpha e^{-\beta\left(g-g_{0}\right)}\right),
$$

where $\alpha$ and $\beta$ are trimming parameters and $g_{0}$ is the reference gap. (A simple alternative to the use of a barrier function would be to define geometrical limits and to "saturate" the position of the profile points if necessary $[8,15]$.

\section{Parametrization of the pole shape}

The pole shape can be simply parametrized by deviations from a reference hyperbolic profile: $X_{K}=x_{k}+\xi_{k}$ and $Y_{K}=y_{k}+\psi_{k}$, where $1 \leq k \leq K$ and $y_{k}=r_{0}{ }^{2} /\left(2 x_{k}\right)$. Here, the parameters $\left(\xi_{k}, \psi_{k}\right)$ give the deviation of the profile to the reference shape. This parametrization has some limitations. The discretization depends on the number of points to optimize, and a smooth shape would imply the optimization of a large number of points. But if the points are close to each other, the displacement of two neighboring points will have almost the same signature on the cost function: the problem is ill conditioned. This difficulty can be solved by expressing the $\left(\xi_{k}, \psi_{k}\right)$ as a sum of smooth functions. Legendre polynomials have been used in the present work. The pole shape parameters are expressed as

$$
\begin{aligned}
\xi_{k} & =\sum_{l=1}^{L} \alpha_{l} P_{l}\left(2 \frac{k-1}{K-1}-1\right) \\
\psi_{k} & =\sum_{l=1}^{L} \beta_{l} P_{l}\left(2 \frac{k-1}{K-1}-1\right),
\end{aligned}
$$

where $\alpha_{l}$ and $\beta_{l}$ are the pole shape parameters and the $P_{l}$ are the Legendre polynomials. The pole smoothness is handled by the maximum polynomial order $L$. This parametrization and the trivial one described above have also both been used for the study of the mechanical tolerances.

\section{Regularized descent method}

A Gauss-Newton algorithm was used. The optimization problem is linearized by computing the Jacobi matrix, and iterating a few times. Let $\zeta_{n}$ be a parameter of the pole shape, with e.g. $\zeta_{n \leq L}=\alpha_{n}$ and $\zeta_{n>L}=\beta_{n-L}$, and $\boldsymbol{\zeta}=\left(\zeta_{1}, \ldots, \zeta_{2 L}\right)$. The value of the parameters at the kth iteration are 


$$
\begin{aligned}
\boldsymbol{\zeta}_{k+1} & =\boldsymbol{\zeta}_{k}-\left(\mathbf{J}_{k}^{T} \mathbf{J}_{k}\right)^{-1} \mathbf{J}_{k}^{T} \boldsymbol{\varepsilon}_{k} \\
& =\boldsymbol{\zeta}_{k}-\mathbf{J}_{k}^{+} \boldsymbol{\varepsilon}_{k} .
\end{aligned}
$$

Here, $\mathbf{J}_{k}$ is the Jacobi matrix at iteration $k, \mathbf{J} k^{T}$ is the transpose of $\mathbf{J}_{k}$ and $\mathbf{J}_{k}{ }^{+}$is its pseudoinverse. In practice, the $\mathbf{J}_{k}$ matrix is ill conditioned and the algorithm diverges rapidly. This issue was overcome by using a singular value decomposition of the matrix $\mathbf{J}_{k}$ :

$$
\begin{aligned}
\mathbf{J}_{k} & =\mathbf{U} \Sigma \mathbf{V}^{T} \\
& =\sum_{i} \mathbf{u}_{i} \sigma_{i} \mathbf{v}_{i}{ }^{T},
\end{aligned}
$$

where $\sigma_{1} \geq \sigma_{2} \geq \cdots \geq 0$ are the singular values of $\mathbf{J}_{k}$, $\mathbf{u}_{i}^{T} \mathbf{u}_{j}=\delta_{i j}$ and $\mathbf{v}_{i}^{T} \mathbf{v}_{j}=\delta_{i j}$. The pseudoinverse is computed according to $\mathbf{J}_{k}{ }^{+}=\mathbf{V} \Sigma^{+} \mathbf{U}^{T}$ where $\Sigma^{+}$is a diagonal matrix with diagonal elements $1 / \sigma_{i}$ if $\sigma_{i} / \sigma_{1} \geq r$ and zero elsewhere. Good results were obtained with $10^{-3} \leq r \leq 10^{-2}$.

A regularized pseudoinverse of the matrix $\mathbf{J}$ can be computed by truncation of the list of its singular values: $\mathbf{J}^{+}=\mathbf{V} \Sigma^{+} \mathbf{U}^{\mathbf{T}}$ where $\Sigma^{+}$is a diagonal matrix with diagonal elements $1 / \sigma_{i}$ if $\sigma_{i} / \sigma_{1}>r$ and zero elsewhere. In practice, good pole shape optimization results are obtained with $10^{-3}<r<10^{-2}$.

\section{Optimization results}

The shape optimization routine was run on the ESRF cluster with ten CPUs. Good results were obtained after 15 minutes (i.e. 150 minutes with one CPU) and five iterations [16], which is pretty fast for the optimization of a 3D model.

Figure 9 and Table IV show two pole profiles obtained with different optimization parameter settings. At nominal current, the field quality is within the specifications and is almost the same for both profiles (Table V).

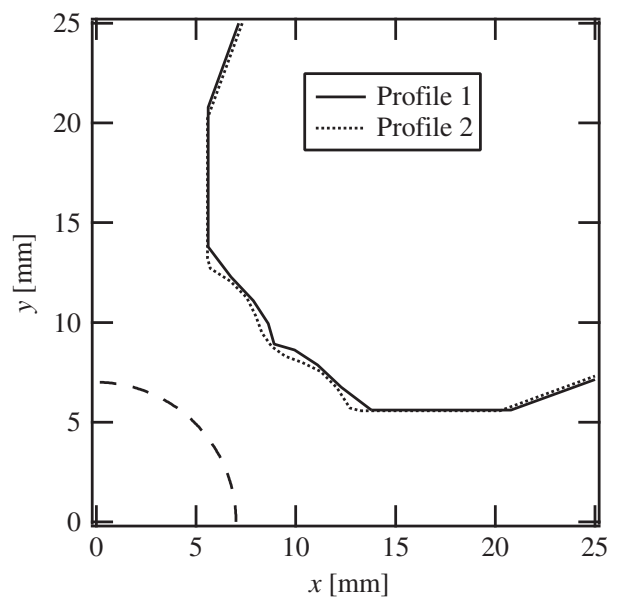

FIG. 9. Pole shapes obtained with different settings of the optimization algorithm. The dashed arc indicates the $7 \mathrm{~mm}$ radius GFR.
TABLE IV. Coordinates $\left(X_{i}, Y_{i}\right)$ of the contour of the two profiles.

\begin{tabular}{lc}
\hline \hline Profile 1 & Profile 2 \\
\hline$(5.611,13.781)$ & $(5.573,13.231)$ \\
$(6.756,12.272)$ & $(5.708,12.706)$ \\
$(7.872,11.081)$ & $(6.717,12.061)$ \\
$(8.611,9.939)$ & $(7.535,11.239)$ \\
$(8.910,8.910)$ & $(7.990,10.332)$ \\
& $(8.309,9.478)$ \\
& $(8.799,8.799)$ \\
\hline \hline
\end{tabular}

TABLE V. Systematic higher order multipoles at $7 \mathrm{~mm}$ radius.

\begin{tabular}{lcc}
\hline \hline & Profile 1 & Profile 2 \\
\hline$b_{6}$ & $-5 \times 10^{-5}$ & $-5 \times 10^{-5}$ \\
$b_{10}$ & $5 \times 10^{-5}$ & $8 \times 10^{-5}$ \\
$b_{14}$ & $-5 \times 10^{-5}$ & $-8 \times 10^{-5}$ \\
$b_{18}$ & $5 \times 10^{-5}$ & $1 \times 10^{-5}$ \\
\hline \hline
\end{tabular}

Figure 10 shows the evolution of the 12-pole with the excitation current. The $b_{6}$ of the quadrupole with sharp poles (Profile 1) is almost independent of the current. This is not the case with Profile 2: this magnet has been optimized at a given excitation, and the iron saturation makes the gradient homogeneity unacceptable at any other current. For current ranging from 10 to $130 \mathrm{~A}$ (90 A nominal), the gradient inhomogeneity is $10^{-4} \leq \Delta G / G \leq$ $4 \times 10^{-4}$ with Profile 1 and $210^{-4} \leq \Delta G / G \leq 22 \times 10^{-4}$ with Profile 2 . Figure 11 shows the magnetization in the pole. As expected, the outer parts of the pole saturate at lower excitation than the pole shapes: this generates the 12-pole term. This 12-pole term is partially compensated by the saturation of the spike located in the central part of the pole.

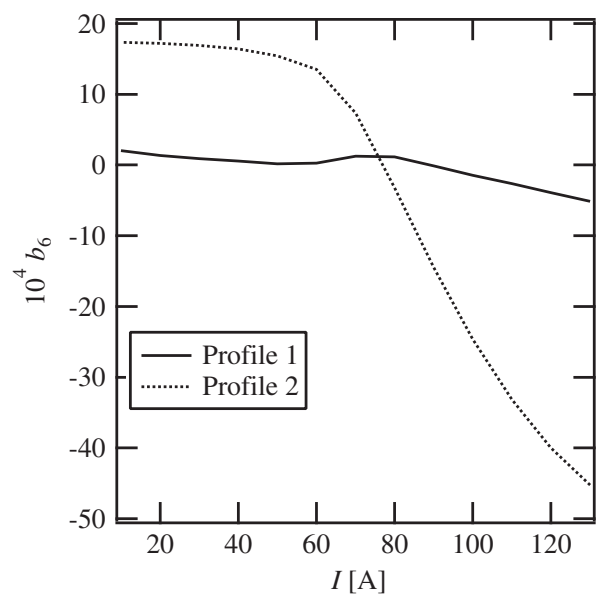

FIG. 10. 12-pole term vs current, for Profile 1 and Profile 2. Reference radius: $7 \mathrm{~mm}$. 

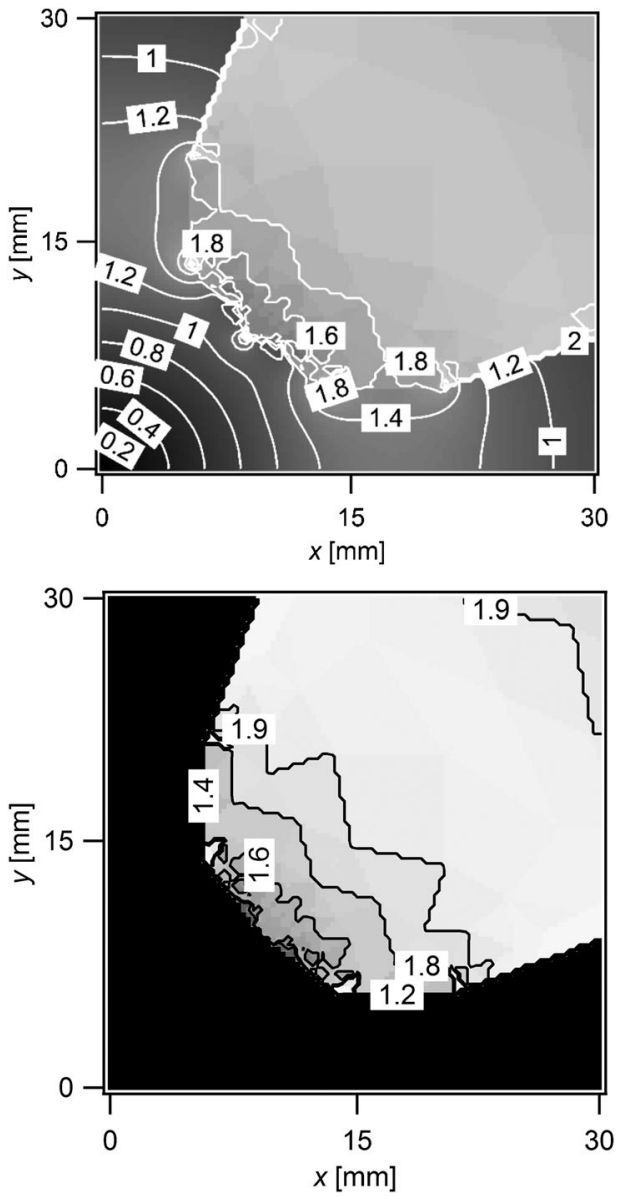

FIG. 11. Pole field $B$ (top) and magnetization $\mu_{0} M$ (bottom) at 90 A, with Profile 1. All values are in [T].

It is a common practice to drill a hole above the pole profile, all along the magnet [17]. The saturation of the central part of the pole is increased by the hole and the effect is similar to the 12-pole compensation scheme described above. The correction presented here has the following advantages: it is compatible with solid iron magnets, and we add magnetic material instead of drilling a hole-thus we can expect a higher field and gradient.

Figure 12 shows the excitation curve of the magnet. At nominal current, the magnet is clearly saturated: the gradient is $14 \%$ below its value interpolated from the linear part of the curve. This is not a big issue, because (i) the sensitivity of the field quality to the current is low and (ii) the power consumption is low due to the small bore radius and the rather low current density.

\section{E. Mechanical tolerances}

High gradient quadrupoles are more sensitive to mechanical errors than lower gradient magnets due to their reduced aperture, as shown in Eqs. (8) and (9). The mechanical tolerances that can be achieved on a magnet series depends on the manufacturing method, on the quality

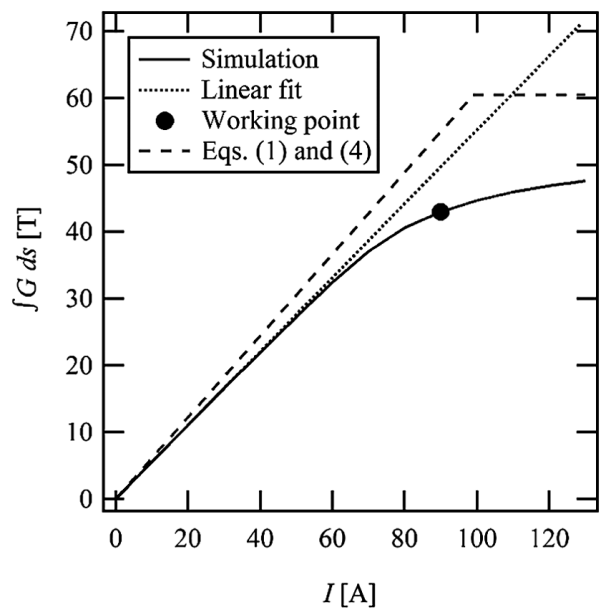

FIG. 12. Excitation curve of the high gradient quadrupole. The results obtained from the analytic model [Eqs. (1) and (4)] are given assuming a $493 \mathrm{~mm}$ magnetic length.

control, on the rejection rate, etc. Considering the $0.5 \mathrm{~m}$ length of ESRF-EBS high gradient magnets, it is assumed that a tolerance of $\pm 0.040 \mathrm{~mm}$ (including pole shapes and pole locations) can be reached with a reasonable effort but $\pm 0.020 \mathrm{~mm}$ is much more difficult and expensive. The impact of the mechanical tolerances on the gradient errors, and finally on the beam dynamics, has been studied in detail.

The impact of some mechanical errors have been studied analytically by Halbach $[18,19]$. A numerical approach is presented here. Two types of random mechanical errors have been considered: random errors on the pole profiles, and random errors on the positions of the poles. The effect of the displacement of the magnetic center is neglected here, assuming that the magnets are magnetically measured and fiducialized.

Using the notations introduced in Sec. IIC 2, the imperfect pole $i$ is described as $\left(X_{k}+\delta x_{k i}, Y_{k}+\delta y_{k i}\right)$. The pole shape errors of a magnet are characterized by the random vector $\left(\delta x_{S 11}, \ldots, \delta x_{S K 4}, \delta y_{S 11}, \ldots, \delta y_{S K 4}\right)$ while the pole displacements are modeled by $\delta x_{D k i}=\delta x_{D i}$ and $\delta y_{D k i}=\delta y_{D i}$, i.e. $\delta w_{k i}=\delta w_{S k i}+\delta w_{D i}$ with $w=\{x, y\}$. One may argue that independent values for the $\delta x_{k i}$ and the $\delta y_{k i}$ would consist of adding a white random noise to the pole shape, which does not represent accurately the machining errors. Indeed, the pole shape errors can be modeled by a sum of functions with random coefficients, as indicated in the Sec. II C 2. For the same mechanical tolerances, it is expected that a "smoother" error profile would lead to an increase of the lower order multipoles and a decrease of the higher order multipoles. Only the "white noise" error case will be presented in this paper. In this case, it is assumed that the errors are distributed according to uniform distributions $\delta W_{S} \sim U(-\mathrm{tol} / 2, \mathrm{tol} / 2)$ and $\delta W_{D} \sim U(-$ tol $/ 2$, tol $/ 2)$. The standard deviations $\sigma\left(a_{n}\right)$ and $\sigma\left(b_{n}\right)$ of the multipole coefficients are then estimated 
TABLE VI. Random multipoles computed for tol $=0.040 \mathrm{~mm}$ and expressed at $7 \mathrm{~mm}$, for the two profiles plotted in Fig. 9.

\begin{tabular}{lcc}
\hline \hline & Profile 1 & Profile 2 \\
\hline$\left[\sigma\left(a_{3}\right)+\sigma\left(b_{3}\right)\right] / 2$ & 0.00052 & 0.00052 \\
{$\left[\sigma\left(a_{4}\right)+\sigma\left(b_{4}\right)\right] / 2$} & 0.00026 & 0.00021 \\
{$\left[\sigma\left(a_{5}\right)+\sigma\left(b_{5}\right)\right] / 2$} & 0.00013 & 0.00011 \\
{$\left[\sigma\left(a_{6}\right)+\sigma\left(b_{6}\right)\right] / 2$} & 0.00007 & 0.00006 \\
\hline \hline
\end{tabular}

with a Monte Carlo method. A set of imperfect magnets is generated assuming the above error distributions, and the multipole content of these imperfect magnets is used to estimate $\sigma\left(a_{n}\right)$ and $\sigma\left(b_{n}\right)$. The random errors expected for the optimized pole profiles obtained in the previous paragraph are given in Table VI.

Figure 13 shows the contribution of the pole shape and the pole displacement errors, assuming equal tolerances of both error types (in practice, for $\pm 0.040 \mathrm{~mm}$ global tolerances, the pole shape errors are expected to be smaller than $\pm 0.020 \mathrm{~mm}$ ). The contributions of the two types of errors are similar for the low order multipoles, then the multipole content is dominated by the pole shapes errors. It should be noted that the 10-pole and 18-pole are induced by pole shape errors only.

The impact of these random multipole errors on the electron beam dynamics has been studied by tracking simulations. The dynamic aperture and the beam lifetime has been computed for different values of tolerance (Table VII). The effect of increasing the mechanical tolerances from $\pm 0.020 \mathrm{~mm}$ to $\pm 0.040 \mathrm{~mm}$ is almost negligible; this means that the aperture and the lifetime are dominated by other errors, namely alignment errors and main multipole strength errors [20].

Following these results, the mechanical tolerances of the series magnets was set to $\pm 0.040 \mathrm{~mm}$. Considering the

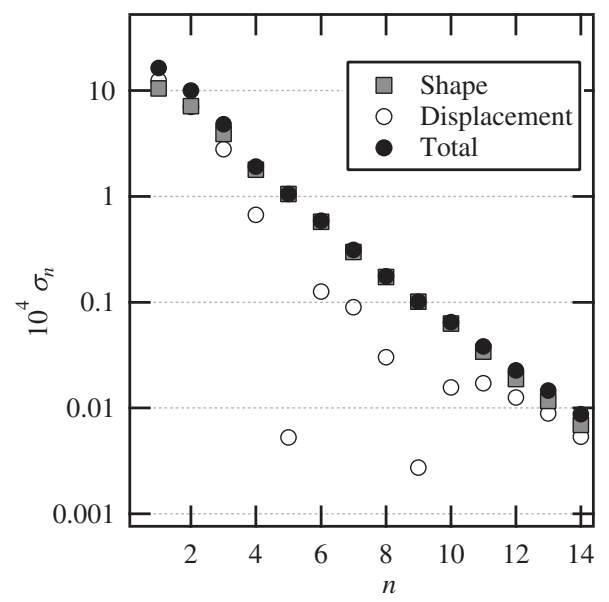

FIG. 13. Standard deviation of the multipoles $\sigma_{n}=\left[\sigma\left(a_{n}\right)+\sigma\left(b_{n}\right)\right] / 2$, expressed at $7 \mathrm{~mm}$. The tolerances are $\pm 0.020 \mathrm{~mm}$ for the shape tolerances, $\pm 0.020 \mathrm{~mm}$ for the pole displacements and $\pm 0.040 \mathrm{~mm}$ for the complete magnet.
TABLE VII. Impact of mechanical tolerances on beam lifetime and dynamic aperture.

\begin{tabular}{lccc}
\hline \hline Tolerances & {$[\mathrm{mm}]$} & \pm 0.020 & \pm 0.040 \\
Beam lifetime & {$[\mathrm{h}]$} & $22.5 \pm 1.9$ & $22.3 \pm 2.2$ \\
Dynamic aperture & {$[\mathrm{mm}]$} & $8.6 \pm 0.8$ & $8.1 \pm 0.9$ \\
\hline \hline
\end{tabular}

$0.5 \mathrm{~m}$ length of the magnet, this is a standard tolerance that can be reached with classical mechanical methods like lamination stacking or machining. However, a prototype with $\pm 0.020 \mathrm{~mm}$ tolerance was built for exploring the technology limitations. The measurements done on this prototype are presented in Sec. II F.

\section{F. Longitudinal field and fringe field}

Using the notations introduced in Appendix, the tangential component of the $3 \mathrm{D}$ field multipole terms are given by

$$
B_{\theta n p}=2 \rho_{0}{ }^{n-1+2 p} k_{n p} \cos n \theta
$$

in the case the $k_{n p}$ are purely imaginary numbers, i.e. there is no skew component. Figure 14 shows the evolution of the integral of first multipoles along the quadrupole. It indicates the $b_{6}$ term is mainly an extremity effect that is not perfectly compensated by the pole shape, while the $b_{10}$ and the $b_{14}$ are induced by the pole shape (or, said differently, by the large gap between the poles). One should notice the agreement between Table V and Fig. 14.

The profile of the poles have been optimized considering only the integrated field multipoles. The first $n=2$ terms, with quadrupole symmetry, are shown in Fig. 15. The contribution of the terms $p>0$ to the integral of the field along a straight line is null. However, these terms generate a small bump, which may have an impact on the beam dynamics. In the horizontal symmetry plane, the field of a normal quadrupole writes

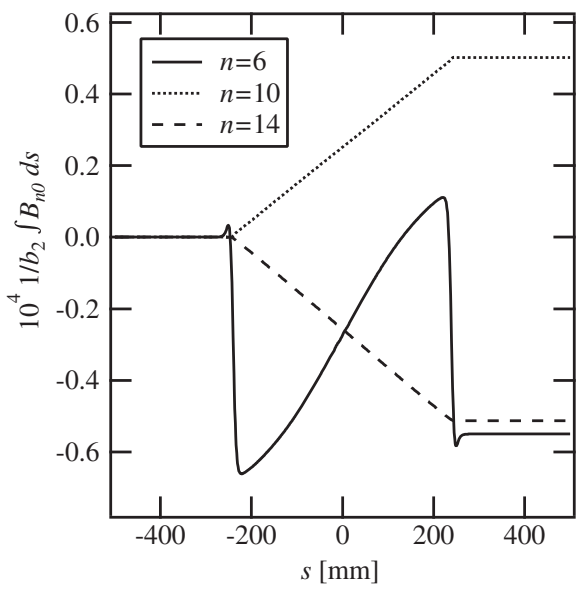

FIG. 14. Simulated integrated field $\int_{-\infty}^{s} \boldsymbol{B}_{n 0} d \nu$ normalized by the integral of the quadrupole term. 


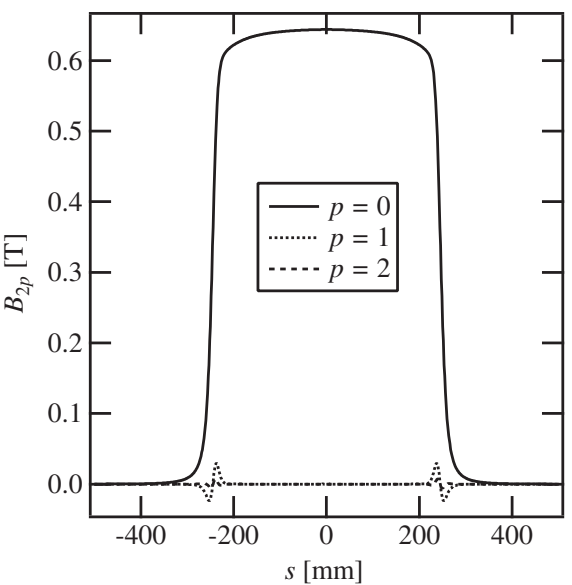

FIG. 15. Simulated field multipoles with quadrupole symmetry: $\boldsymbol{B}_{\theta 20} \propto \rho \cos (2 \boldsymbol{\theta}), \boldsymbol{B}_{\theta 21} \propto \rho^{3} \cos (2 \boldsymbol{\theta})$ and $\boldsymbol{B}_{\theta 22} \propto \rho^{5} \cos (2 \boldsymbol{\theta})$.

$B_{\rho}=0$

$B_{\theta}=2 k_{20}(s) \rho \cos (2 \theta)+2 k_{21}(s) \rho^{3} \cos (2 \theta)+\cdots$.

The amplitude of the bump generated by the third order term in Eq. (16) is

$$
\begin{aligned}
\Delta x(s) & =-\frac{2 e}{m_{e} c \gamma} \int_{-\infty}^{s} \int_{-\infty}^{v} k_{21}(u) \rho^{3} d u d v \\
& =-\frac{e}{6 m_{e} c \gamma} k_{20}(s) \rho^{3} .
\end{aligned}
$$

This induces an error on the integrated magnetic field:

$$
\begin{aligned}
\int B_{\theta} d s & \approx b_{2} \frac{\rho}{\rho_{0}} \cos 2 \theta+\left(\int k_{20} \Delta x d s\right) \rho^{3} \cos 2 \theta \\
& \approx b_{2} \frac{\rho}{\rho_{0}} \cos 2 \theta-\left(\frac{e}{6 m_{e} c \gamma} \int k_{20}^{2} d s\right) \rho^{3} \cos 2 \theta
\end{aligned}
$$

The effect of the fringe field depends on the longitudinal profile $k_{20}$ of the gradient. For the same integrated gradient, the errors are $2 \pi^{1 / 2} \approx 3.5$ times higher for a rectangular profile, compared to a Gaussian profile.

The same arguments applied to the radial field lead to an angular excursion $\Delta \theta$ and to a similar expression of the integrated field:

$$
\int B_{\rho} d s \approx b_{2} \frac{\rho}{\rho_{0}}\left(1+\frac{e \rho^{2} \int k_{20} d s}{3 m_{e} c \gamma} \cos 2 \theta\right) \sin 2 \theta .
$$

For the present design, the integrated field error induced by the fringe field is

$$
\begin{aligned}
& \frac{\Delta B_{\theta}}{B_{\theta}} \approx 2 \times 10^{-7}, \\
& \frac{\Delta B_{\rho}}{B_{\rho}} \approx 1.8 \times 10^{-5} .
\end{aligned}
$$

These two errors are low, but the radial component is not far below the systematic errors induced by the pole shapes.

The approach used here is rather qualitative and should not be used for accurate beam dynamics computations. Hamiltonian descriptions of the fringe field effects are available in the literature, see for instance [21]. However, we believe the present description is quite intuitive and easy to understand. In a relatively recent paper, Baartman developed a quadrupole with longitudinally shaped poles for decreasing the fringe field aberrations [22]. Equation (20) shows extruded poles are adequate for long, high gradient quadrupoles to be used for focusing $6 \mathrm{GeV}$ electrons.

\section{PROTOTYPE AND MEASUREMENTS}

A $86 \mathrm{~T} / \mathrm{m}$ nominal gradient magnet prototype has been built and measured. The main parameters of this magnet are given in Table II. Figure 16 shows the prototype installed on a measurement bench. It was manufactured by industry in a build-to-print approach. The mechanical tolerances were set to $\pm 0.020 \mathrm{~mm}$. The yoke was made with four massive poles and two massive spacers on the sides; it was foreseen to install $\mathrm{C}$-shaped spacers on the final design in order to allow space for the X-ray beam ports. The magnet was made in AISI 1010 steel. The four poles, with the spacers installed, were machined independently with a five axis computed numerical control machine. Better results would be obtained with wire erosion of the assembled yoke, but the magnet length was not compatible with this type of machining. It should be mentioned that the assembled magnet was within the mechanical tolerances, but cost and the machining time were prohibitive for series production.

The magnet was measured with a stretched wire bench [23]. The measurement method is simple: a wire is moved along circular trajectories within the magnet aperture, and the induced voltage is measured and analyzed. The main

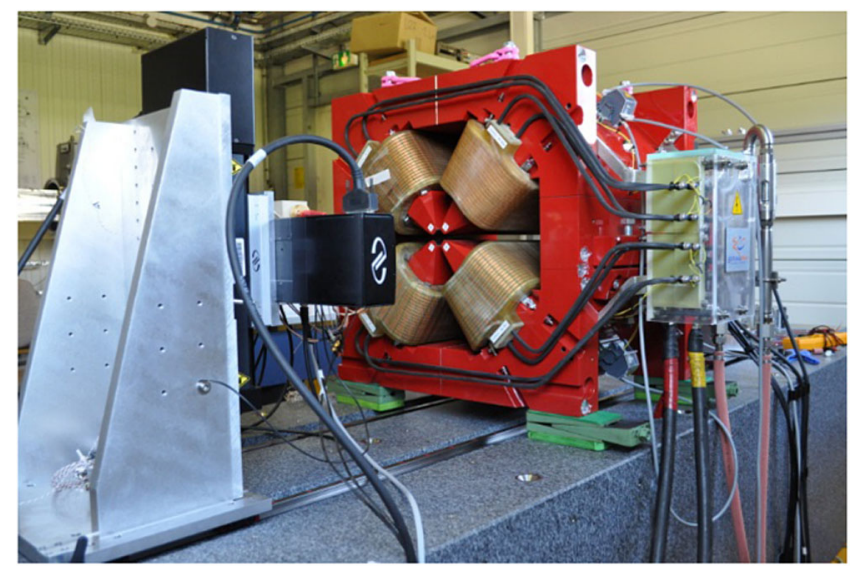

FIG. 16. High gradient quadrupole prototype installed on a stretched wire measurement bench [4]. 
difficulty in this measurement method is the accuracy of the motion, which is improved by an interferometric measurement of the linear stage scales and by a fine-tuning of their angle. Stretched wire benches are suitable for any kind of integral field measurement: field and gradient strength, higher order multipoles and magnetic axis characterization.

The measurement uncertainties can be estimated statistically (i.e. the uncertainties are type A uncertainties according to the guide to the expression of uncertainty in measurement [24]). The repeatability of the measurements is obtained with usual methods: the same measurements are repeated several times, which enables the estimation of a standard deviation. The systematic errors can also be estimated. The range of the linear stages used for positioning the wire is a few times larger than the magnet aperture: measuring the same magnet with different transverse and vertical offsets leads to different wire position errors. The standard deviation of the systematic errors has been estimated with this method, and these errors are 1 order of magnitude higher than the repeatability errors. The error bars shown in the graphs have been estimated with this method.

The magnet gradient is shown in Fig. 17. The $86 \mathrm{~T} / \mathrm{m}$ nominal gradient is reached at $87 \mathrm{~A}$ and a $96 \mathrm{~T} / \mathrm{m}$ gradient was measured at $110 \mathrm{~A}$, which was the maximum current of the power supply. (One may notice that the gradient indicated in Fig. 1 is $91 \mathrm{~T} / \mathrm{m}$, and not $86 \mathrm{~T} / \mathrm{m}$, which was the value at the time the prototype was designed. The magnet has been redesigned for a higher gradient and the measurements presented in Fig. 17 make us confident in the simulations.)

The measured higher order multipoles of the high gradient prototype are shown in Fig. 18. The measured normal multipoles agree with the simulation, except for the sextupole term, which was not allowed by the symmetry of the model. According to Fig. 13, the 10-pole terms should be zero in the case the poles are identical. The observation

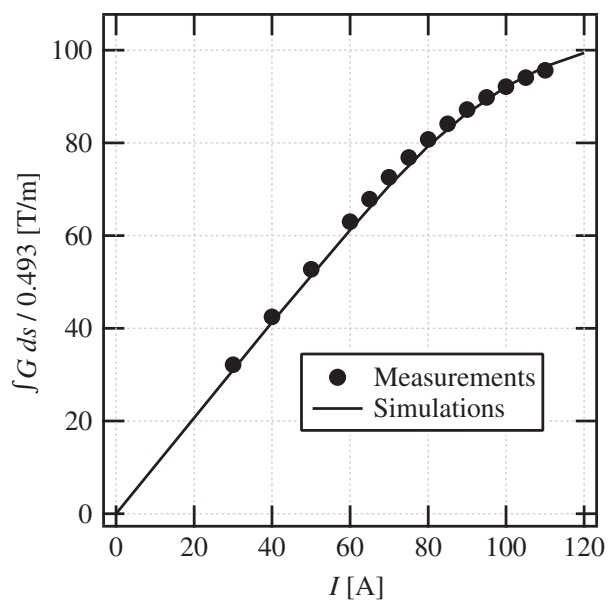

FIG. 17. Quadrupole gradient normalized by a $493 \mathrm{~mm}$ magnetic length. The gradient has been measured with circular measurements at $12.4 \mathrm{~mm}$ radius.
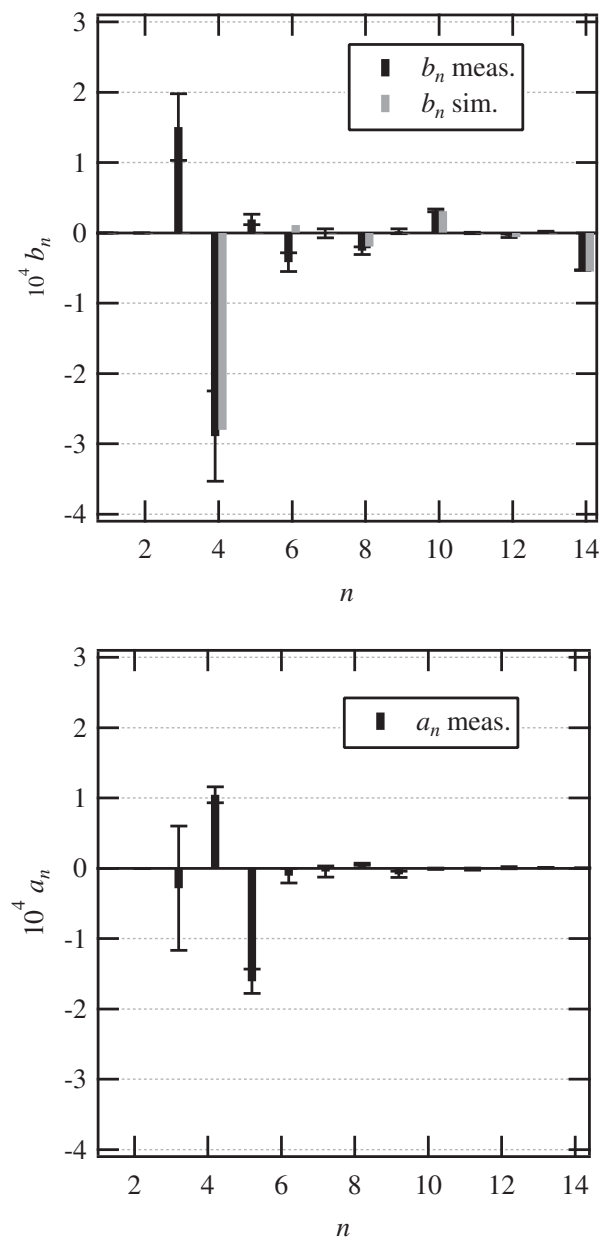

FIG. 18. Higher order multipoles of the high gradient quadrupole prototype at $90 \mathrm{~A}$ and expressed at $7 \mathrm{~mm}$ radius. Circular measurements at different radii, ranging from $5 \mathrm{~mm}$ up to $12 \mathrm{~mm}$, have been combined for measuring the multipoles. The standard deviations have been computed at only one radius, which is a pessimistic case. The dipole and quadrupole terms are not shown. The simulations are for interpole distances with errors equal to $\pm 0.020 \mathrm{~mm}$ (depending on the pole), with a symmetry that does not allow sextupole terms.

of a non-negligible 10-pole is a signature of the pole profile errors.

\section{CONCLUSION}

High gradient quadrupoles are expected to be installed in the next generation of light sources. The pole tip field of these quadrupoles is higher than usual, leading to a saturation of the yoke. However, this saturation is not so detrimental: the power consumption is kept to reasonable values at the price of the slight increase of transverse dimensions, and the field quality is correct within a wide current range thanks to the optimized shape of the poles.

Due to their small aperture, high gradient quadrupoles have an increased sensitivity to mechanical errors. Random multipole errors have been computed for two 
manufacturing methods: stacking of laminations, leading to $\pm 0.040 \mathrm{~mm}$ tolerances on the magnet assembly, and fine machining of solid iron poles, for which $\pm 0.020 \mathrm{~mm}$ tolerances were assumed. Beam dynamics computations have shown that the electron beam lifetime and the dynamic aperture are not highly affected by the mechanical errors (i.e. they are dominated by other errors: alignment, strength, etc.) and the $\pm 0.040 \mathrm{~mm}$ mechanical tolerances are sufficient for these magnets.

A prototype has been built and measured. The mechanical tolerances of the prototype were specified to $\pm 0.020 \mathrm{~mm}$. The tolerances were reached, but the machining time was long and the cost was not suitable for mass production. It was measured at the ESRF. A $86 \mathrm{~T} / \mathrm{m}$ gradient was obtained at nominal current, in good agreement with the simulation results. The measured field quality was compatible with the mechanical tolerances, assuming the largest tolerable errors are reached.

This work has demonstrated the feasibility of such high gradient quadrupoles for mass production. The scale factors presented in Sec. I have a beneficial impact on the cost: high gradient quadrupoles have a small aperture, so they are compact. The poles are saturated at relatively low amp turns, so the power consumption is reasonable. The procurement of 135 high gradient quadrupoles has started. The specifications allowed both standard lamination stacking, lamination stacking with final machining, and machining of solid iron blocks (the mechanical tolerances is $\pm 0.040 \mathrm{~mm}$ for the series). It should be noted that the best offers were for the machining of solid iron blocks.

\section{APPENDIX: HARMONIC FIELD EXPANSIONS AND FIELD MULTIPOLES}

It is a common practice to express the magnetic field of accelerator magnets as a sum of harmonic functions. Accelerator magnet designers are familiar with 2D circular multipoles. The theory of this simple field expansion, and the notations used in the following of this paper, are briefly covered in the next paragraph. Cylindrical circular multipoles are a useful tool for the investigation of the fringe fields. These multipoles are introduced at the end of this section.

\section{2D circular multipoles}

In accelerator magnets, the region of interest is free of current and magnetic material: the Maxwell equations simplify to $\nabla \cdot \mathbf{B}=0$ and $\nabla \times \mathbf{B}=0$. In $2 \mathrm{D}$, the field components in Cartesian coordinates satisfy the CauchyRiemann equations. The complex field $B=B_{Y}+i B_{X}$ is an analytic function of the complex variable $z=x+i y$, where $x$ (respectively $y$ ) is the horizontal (respectively vertical) coordinate. The Taylor series of the complex field writes, using common magnet design notations,

$$
B=\mathscr{B} \sum_{n=1}^{\infty}\left(b_{n}+i a_{n}\right)\left(\frac{z}{\rho_{0}}\right)^{n-1},
$$

where $\rho_{0}$ is a reference radius and the $b_{n}$ and $a_{n}$ are the so-called normal and skew multipole coefficients. The multipole coefficients are normalized to one for the quadrupole component. If the coordinate system is aligned on the magnetic symmetry plane, one has $\left(a_{2}, b_{2}\right)=(0,1)$. The quadrupole strength is defined as $G=\mathscr{B} / \rho_{0}$.

The multipole coefficients are usually derived from the Fourier transform of a set of field values sampled at equally spaced intervals on a circle of radius $\rho_{0}$. More generally, the complex field at given arbitrary points is linked to the first multipole coefficients by the matrix relation

$$
\mathbf{B}=\mathbf{M}_{\mathbf{C}} \mathbf{C},
$$

where $\quad B_{i}=B\left(z_{i}\right), \quad C_{j}=b_{j}+i a_{j}, \quad M_{C i 1}=\mathscr{B} \quad$ and $M_{C i j>1}=\mathscr{B}\left(z_{i} / \rho_{0}\right)^{j-1}, 1 \leq i \leq M$ and $1 \leq j \leq N$. The multipole coefficients are estimated from a pseudoinverse $\mathbf{M}_{\mathbf{C}}{ }^{+}$of $\mathbf{M}_{\mathbf{C}}$ :

$$
\begin{aligned}
\mathbf{C} & =\left(\mathbf{M}_{\mathbf{C}}{ }^{T} \mathbf{M}_{\mathbf{C}}\right)^{-1} \mathbf{M}_{\mathbf{C}}{ }^{T} \mathbf{B} \\
& =\mathbf{M}_{\mathbf{C}}{ }^{+} \mathbf{B} .
\end{aligned}
$$

This expression of the multipole coefficients has been introduced for magnetic measurements [20] and is also useful for the optimization of magnets with noncircular GFR.

\section{3D circular cylinder multipoles}

The theory of circular cylinder multipoles has been presented many times. A detailed and well written presentation of this field expansion can be found in Davies's paper [25]. The main results are summarized in this paragraph.

The magnetic field derives from a scalar potential because the volume of interest is free of currents and equivalent magnetic charges. The Laplace equation is separable in cylindrical coordinates and the solution writes

$$
\phi=R_{n}(\kappa) I_{n}(\kappa \rho) e^{i n \theta} e^{i(\kappa s+\gamma)}+C_{n} \rho^{n} e^{i n \theta},
$$

where $I_{n}$ is the modified Bessel function of the first kind. A series expansion of the Bessel function and a Fourier transform on the $s$ variable leads to

$$
\phi(\rho, \theta, s)=\sum_{n \geq 0} \sum_{p \geq 0} k_{n p}(s) \rho^{n+2 p} e^{i n \theta} .
$$

The $k_{n p}$ terms in this equation can be computed from a $2 \mathrm{D}$ Fourier transform of a set of field values, sampled on the surface of a reference cylinder [25].

The $k_{n p}$ 's have the following properties (the proofs can be found in the above references). The only terms with 
nonzero integral over $s$ are the terms $k_{n 0}(s)$. Moreover, all of the terms can be deducted from the $p=0$ terms:

$$
k_{n p+1}(s)=\left(-\frac{1}{4}\right)^{p} \frac{n !}{p !(n+p) !} \frac{d^{2 p}}{d s^{2 p}} k_{n 0}(s) .
$$

The radial and tangential field components are computed using $B_{\rho}=-\operatorname{Re}(\partial \phi / \partial \rho)$ and $B_{\theta}=-\operatorname{Re}(1 / \rho \partial \phi / \partial \theta)$. The identification of the $k_{n 0}$ terms with Eq. (A2) leads to

$$
\begin{aligned}
& a_{n}=-n \rho_{0}{ }^{n-1} \int \operatorname{Re}\left[k_{n 0}(s)\right] d s, \\
& b_{n}=-n \rho_{0}{ }^{n-1} \int \operatorname{Im}\left[k_{n 0}(s)\right] d s .
\end{aligned}
$$

[1] J.A. Eriksson et al., The MAX IV synchrotron light source, in Proceedings of the 2nd International Particle Accelerator Conference, San Sebastián, Spain (EPS-AG, Spain, 2011), pp. 3026-3028.

[2] L. Farvacque et al., A low-emittance lattice for the ESRF, in Proceedings of the 4th International Particle Accelerator Conference, IPAC-2013, Shanghai, China, 2013 (JACoW, Shanghai, China, 2013), pp. 79-81.

[3] M. Borland, V. Sajaev, Y. Sun, and A. Xiao, Hybrid sevenbend-achromat lattice for the advanced photon source upgrade, in Proceedings of the 6th International Particle Accelerator Conference, Richmond, USA, 2015 (JACoW, USA, 2015), pp. 1776-1779.

[4] G. Le Bec, J. Chavanne, F. Villar, C. Benabderrahmane, S. Liuzzo, J.-F. Bouteille, L. Goirand, L. Farvacque, J.-C. Biasci, and P. Raimondi, Magnets for the ESRF diffraction limited light source project, IEEE Trans. Appl. Supercond. 26, 4000107 (2016).

[5] P. N'gotta, Développement d'un quadripôle à aimant permanent à usage des accélérateurs de particules, Ph.D. thesis, Université Joseph Fourier, Grenoble, 2015.

[6] J. Chavanne, P. Elleaume, and P. Van Vaerenbergh, The ESRF insertion devices, J. Synchrotron Radiat. 5, 196 (1998).

[7] J. Chavanne, P. Van Vaerenbergh, and P. Elleaume, A 3 T asymmetric permanent magnet wiggler, Nucl. Instrum. Methods Phys. Res., Sect. A 421, 352 (1999).

[8] G. Le Bec and J. Chavanne, Permanent magnet steerers for canted undulators at the ESRF, Nucl. Instrum. Methods Phys. Res., Sect. A 664, 214 (2012).

[9] J. V. Mathew, S. V. L. S. Rao, S. Krishnagopal, and P. Singh, An improved permanent magnet quadrupole design with larger good field region for high intensity proton linacs, Nucl. Instrum. Methods Phys. Res., Sect. A 727, 12 (2013).

[10] P. Elleaume, O. Chubar, and J. Chavanne, Computing 3D magnetic fields from insertion devices, in Proceedings of the Particle Accelerator Conference, Vancouver, BC, Canada, 1997 (IEEE, New York, 1997), pp. 3509-3511.

[11] O. Chubar, P. Elleaume, and J. Chavanne, A threedimensional magnetostatics computer code for insertion devices, J. Synchrotron Radiat. 5, 481 (1998).

[12] K. Halbach, Program of inversion of system analysis and its application to the design of magnets, in MT 2, Oxford, 1967.

[13] A. G. Armstrong, M. Fan, J. Simkin, and C.W. Trowbridge, Automated optimization of magnet design using the boundary integral method, IEEE Trans. Magn. 18, 620 (1982).

[14] H. Siebold, A computer program for optimizing homogeneous fields with iron yoke, IEEE Trans. Magn. 24, 408 (1988).

[15] S. Russenschuck, Field Computation for Accelerator Magnets (Wiley-VCH, Weinheim, 2010).

[16] G. Le Bec, J. Chavanne, and P. N'gotta, Shape optimization for the ESRF II magnets, in Proceedings of the 5th International Particle Accelerator Conference, Dresden, 2014, Germany (JACoW, Germany, 2014), pp. 1232-1234.

[17] Y. Iwashita, A. Noda, T. Shirai, A. Morita, M. Tadokoro, J. Hirota, M. Umezawa, and K. Hiramoto, Novel design for electromagnet with wide excitation range, Jpn. J. Appl. Phys. 38, L895 (1999).

[18] K. Halbach, First order perturbation effects in irondominated two-dimensional symmetrical multipoles, Nucl. Instrum. Methods 74, 147 (1969).

[19] J. Tanabe, Iron Dominated Electromagnets (World Scientific, Singapore, 2005).

[20] S. M. Liuzzo et al., Influence of errors on the ESRF upgrade lattice, in Proceedings of the 6th International Particle Accelerator Conference, Richmond, USA, 2015 (JACoW, USA, 2015), pp. 1426-1428.

[21] R. Baartman, Intrinsic third order aberrations in electrostatic and magnetic quadrupoles, in Proceeding of the Particle Accelerator Conference, Vancouver, BC, Canada, 1997 (IEEE, New York, 1997), pp. 1415-1417.

[22] R. Baartman, Quadrupole shapes, Phys. Rev. ST Accel. Beams 15, 074002 (2012).

[23] G. Le Bec, J. Chavanne, and C. Penel, Stretched wire measurement of multipole accelerator magnets, Phys. Rev. ST Accel. Beams 15, 022401 (2012).

[24] Bureau International des Poids et Mesures, Evaluation of measurement data-Guide to the expression of uncertainty in measurement, 2008.

[25] W. G. Davies, The theory of the measurement of magnetic multipole fields with rotating coil magnetometers, Nucl. Instrum. Methods Phys. Res., Sect. A 311, 399 (1992). 